\title{
A formação de enfermeiros e sua aproximação com os pressupostos das Diretrizes Curriculares Nacionais e da Atenção Básica
}

\author{
Nursing training and their approximation to the assumptions of \\ the National Curriculum Guidelines and Primary Health Care
}

Carinne Magnago (http://orcid.org/0000-0001-8799-3225) ${ }^{1}$

Celia Regina Pierantoni (http://orcid.org/0000-0001-7481-6350) ${ }^{1}$

${ }^{1}$ Instituto de Medicina Social, Universidade do Estado do Rio de Janeiro. R. São Francisco Xavier 524, 7 o andar, Maracanã. 20550-013 Rio de Janeiro RJ Brasil. carinne.mag@gmail.com

\begin{abstract}
This article describes the characteristics of offer for job vacancies, curricular structure and competency profile of students from nursing undergraduate courses, seeking to identify elements that are in line with the assumptions of the $\mathrm{Na}$ tional Curricular Guidelines (NCG) and of the nurses' work in primary health care. Applying a computer-assisted survey by telephone interviews with 94 course coordinators, it was verified: provision of job vacancies distant from the national needs; curricular structure focused on fragmented disciplines in the basic and vocational cycles, in disagreement with recommendations by the NCG; and competency profile potent for the development of actions of promotion, prevention, management and nursing techniques, which are predicted in the scope of general skills and abilities recommended for the exercise of the generalist professional nursing practice, according to the NCG, and compatible with the main demands of primary health care.
\end{abstract}

Key words Higher Education, Nursing, Unified Health System, Curriculum, Primary Health Care
Resumo Este artigo descreve as características da oferta de vagas, da estrutura curricular e do perfil de competências de egressos de cursos de graduação em enfermagem, buscando identificar elementos que se aproximam com os pressupostos das Diretrizes Curriculares Nacionais (DCN) e da atuação profissional do enfermeiro na atenção básica. Aplicando-se um survey por entrevistas telefônicas assistidas por computador com 94 coordenadores de cursos, verificou-se: oferta de formação distante das necessidades nacionais; estrutura curricular centrada em disciplinas fragmentadas nos ciclos básico e profissionalizante, destoando do recomendado pelas DCN; e perfil de competências potente para o desenvolvimento de ações de promoção, prevenção, gerenciamento e técnicas de enfermagem, as quais são previstas no escopo de competências e habilidades gerais preconizadas para o exercício profissional generalista da enfermagem, segundo as DCN, e compatíveis com as principais demandas assistenciais da atenção básica.

Palavras-chave Educação superior, Enfermagem, Sistema Único de Saúde, Currículo, Atenção primária à saúde 


\section{Introdução}

A profissão surge como instrumento de resposta às necessidades coletivas historicamente apresentadas. Assim, tal como as demais profissões da área da saúde, a enfermagem teve, na sua constituição, influência das demandas assistenciais da população e dos movimentos políticos e sociais por saúde, bem como das manifestações hegemônicas de poder ${ }^{1}$. Desta forma, a construção da profissão e, por conseguinte, do ensino de enfermagem, perpassa às mudanças experimentadas no percurso histórico da saúde ${ }^{2}$.

Na década de 1920, o ensino e a atuação da enfermagem visavam predominantemente à saúde pública, por meio de práticas primárias de saúde preventivas realizadas fora do ambiente hospitalar e vinculadas ao Estado. Na década de 1940, sofreram repercussões da consolidação da indústria e surgimento de grandes hospitais, centrando-se no modelo clínico de prática fragmentada, voltado para a área hospitalar ${ }^{3}$.

Nessa conjuntura, o currículo de ensino da enfermagem passa a focar as clínicas especializadas, dando foco à doença e à cura, e a saúde pública deixa de ser matéria obrigatória ${ }^{4}$. No governo militar, observou-se uma diminuição dos gastos estatais em saúde pública, com redução dos programas dirigidos ao controle das doenças de massa e viabilização do crescimento do complexo médico-industrial, consolidando o modelo de saúde biomédico, centrado no hospital ${ }^{5}$.

Na década de 1980 ocorrem mudanças políticas no País, acarretando uma série de propostas de reformas na organização dos serviços de saúde e mercado de trabalho do enfermeiro, com reflexos na sua formação ${ }^{6}$. Após a criação do Sistema Único de Saúde (SUS), levantou-se debates acerca do perfil de competências que os enfermeiros deveriam assumir, culminando na aprovação das Diretrizes Curriculares Nacionais para os Cursos de Graduação em Enfermagem (DCENF), em $2001^{7}$.

As DCENF dispõem sobre o perfil do enfermeiro que deveria, a partir de agora, ter formação generalista, humanista, crítica e reflexiva, além de elucidar os conteúdos curriculares que deveriam compreender as Ciências Biológicas e Sociais da Saúde, as Ciências Humanas e as Ciências de Enfermagem, que compreendem os fundamentos, a administração, o ensino e a assistência de enfermagem ${ }^{7}$

Considerando as especificidades do SUS e sua priorização às ações de promoção e prevenção, a atenção básica foi considerada nível primário e fundamental para toda a organização desse sistema. Nessa perspectiva, as DCENF trazem definição robusta na abordagem de competências específicas para atuação nesse nível de atenção, destacando-se a atuação em equipe multiprofissional, a integralidade da atenção, a ênfase na promoção à saúde, o foco nos indivíduos, família e comunidade e competências para o planejamento em saúde. Nessa lógica, a inserção dos alunos na comunidade e em diferentes espaços e níveis de atenção à saúde e a criação de vínculo são pressupostos importantes para a formação de enfermeiros ${ }^{7}$.

As necessidades de saúde cada vez mais dinâmicas e complexas, marcadas especialmente pelo surgimento e reemergência de doenças, além do envelhecimento populacional que tem aumentado a prevalência das doenças crônicas, demandam uma atenção básica mais resolutiva e, por conseguinte, enfermeiros de perfil generalista e competente para o trabalho em equipe interdisciplinar e coletivo, capaz de desenvolver ações efetivas, especialmente de cunho preventivo ${ }^{8,9}$.

Pelo exposto, este estudo objetivou descrever as características da oferta e da estrutura curricular e o perfil de competências de egressos de cursos de graduação em enfermagem do Brasil, buscando identificar elementos que se aproximam com os pressupostos das DCENF e da atuação profissional do enfermeiro na Atenção básica.

\section{Método}

Trata-se de um estudo nacional, descritivo e quantitativo, desenvolvido entre dezembro de 2015 e junho de 2016. Os dados foram coletados por survey conduzido com gestores de cursos de graduação em enfermagem (coordenadores e diretores), mediante Entrevista Telefônica Assistida por Computador (ETAC).

O universo da pesquisa correspondeu ao número de cursos de graduação em enfermagem no Brasil ( $\mathrm{n}=868)$, conforme Censo da Educação Superior $2013^{10}$. Para determinação da amostra, procedeu-se cálculo para amostra aleatória simples, considerando-se $90 \%$ de nível de confiança e 6\% de margem de erro, resultando em 154 cursos.

Aleatoriamente as coordenações de curso foram sendo contactadas, visando alcançar o número amostral calculado. Após seis meses, 357 cursos foram acionados: mais de dez tentativas foram efetuadas com 130 deles, sem sucesso; 129 coordenadores se recusaram a participar do estu- 
do ou reagendaram a entrevista por mais de cinco vezes; 94 entrevistas foram completadas; e 04 cursos já não se encontravam mais em atividade. Em razão do tempo prolongado do período de coleta de dados, optou-se por finalizá-la mesmo não se tendo alcançado a amostra final almejada, que foi composta por 94 cursos de graduação, representando uma taxa de $61,0 \%$ da amostra calculada e de 10,8\% da população da pesquisa.

Para execução da ETAC, um questionário semiestruturado foi confeccionado em formulário eletrônico, com questões relativas à identificação do entrevistado, localização, oferta de vagas e estrutura curricular. Ademais, no intuito de compreender a preparação profissional empiricamente oferecida aos alunos, foram citadas diferentes competências e habilidades, classificadas como ações de saúde, ações gerenciais, procedimentos técnicos e procedimentos de apoio diagnóstico e prescrições, para as quais os entrevistados deveriam, por meio de uma escala de pontos, concordar (3), concordar parcialmente (2) ou discordar (1) quanto ao curso preparar os alunos para executá-las. Cabe ressaltar que algumas delas não fazem parte do escopo de prática legal da enfermagem, mas foram adicionadas com o objetivo de investigar se o processo formativo contempla a ampliação do campo de atuação dos enfermeiros. As competências e habilidades foram elencadas com base nas DCENF ${ }^{7}$, em documentos ministeriais da saúde que descrevem atribuições profissionais para os membros das equipes de atenção básica e em resoluções do Conselho Federal de Enfermagem que dispõem sobre atividades de enfermagem regulamentadas ${ }^{11}$.
Os dados foram tabulados e tratados estatisticamente no Statistical Package for the Social Sciences (SPSS) 21.0. Foram empregadas análises descritivas, com cálculo de frequências, percentuais e média ponderada, e análise inferencial pelo coeficiente de concordância de Kendall $\left(\mathrm{W}_{\mathrm{a}}\right)$ para avaliar a associação de respostas entre os respondentes, cujos valores podem variar de 0 a 1 (0-100\%). Considerou-se significância estatística de $5 \%$.

Seguindo a determinação da Resolução 466, de 12 de dezembro de 2012, o projeto do estudo foi submetido à avaliação de Comitê de Ética em Pesquisa.

\section{Resultados}

\section{Perfil dos entrevistados}

A amostra de entrevistados $(n=94)$ foi composta majoritariamente por mulheres $(n=79$; $84 \%)$; enfermeiros $(n=89 ; 94,7 \%)$; mestres $(n=53 ; 56,4 \%)$ e doutores $(n=33 ; 35,1 \%)$. Registrou-se idade média de 47 anos e tempo médio de formação e no cargo de gestão de 21,7 e 4,5 anos, respectivamente.

\section{Localização e natureza jurídica dos cursos}

A maioria dos cursos se localiza na região $\mathrm{Su}$ deste e em municípios não capitais com mais de 100 mil habitantes, conforme se verifica na Tabela 1. Quanto à natureza jurídica, a maior parte dos cursos é proveniente de instituições de ensi-

Tabela 1. Cursos de graduação em enfermagem da amostra, segundo localização por região e porte populacional dos municípios. Brasil, 2015-2016 ( $\mathrm{n=94).}$

\begin{tabular}{lccrc}
\hline \multicolumn{1}{c}{ Localização dos cursos } & \multirow{2}{*}{ Privadas $(\mathbf{n}=\mathbf{7 0})$} & Públicos $(\mathbf{n}=\mathbf{2 4})$ & \multicolumn{2}{c}{ Total } \\
\cline { 4 - 5 } Regiões & 5 & & $\mathbf{N}$ & \% \\
$\quad$ Norte & 13 & 7 & 5 & 5,3 \\
Nordeste & 38 & 3 & 41 & 43,3 \\
Sudeste & 8 & 10 & 18 & 19,1 \\
Sul & 6 & 4 & 10 & 10,6 \\
Centro-Oeste & & & & \\
Porte populacional (habitantes) & 28 & 6 & 34 & 36,2 \\
Capitais & 25 & 11 & 36 & 38,3 \\
Mais de 100 mil (não capitais) & 12 & 5 & 17 & 18,1 \\
Mais de 50 até 100 mil & 5 & 2 & 7 & 7,4 \\
Mais de 20 até 50 mil & & & &
\end{tabular}

Fonte: Dados da pesquisa. 
no superior privadas $(\mathrm{n}=70 ; 74,5 \%)$; as públicas somaram $24(25,5 \%)$.

\section{Oferta de vagas}

Os cursos ofertam, em média, 124 vagas ao ano, com média de preenchimento de $80 \%$, o que significa dizer que $20 \%$ das vagas ficam ociosas. Os privados ofertam, em média, 146 vagas/ano, das quais $75,3 \%$ são preenchidas; já das 60 vagas/ ano ofertadas, em média, pelos cursos públicos, 93,6\% são preenchidas.

\section{Estrutura curricular dos cursos}

A maioria dos entrevistados $(n=43 ; 45,7 \%)$ declarou que não houve consultoria externa para a elaboração do Projeto Político Pedagógico (PPP), porém, há que se considerar que uma parte dos entrevistados $(n=15)$ não soube responder a esta questão. Do total, 76 (80,9\%) PPP sofreram a última modificação/atualização no período 20132016.

Em relação à estruturação pedagógica, foi perguntado aos gestores qual a ênfase de formação do curso em relação a algumas áreas de atuação dos enfermeiros e, em resposta, o entrevistado deveria classificar cada área em uma escala crescente de 1 (menor ênfase) a 6. As médias ponderadas indicaram a atenção básica como a de maior ênfase $(5,6)$, seguida pela atenção hospitalar $(4,8)$, gestão de serviços $(3,9)$, atenção especializada/ambulatorial $(3,6)$, pesquisa $(3,2)$ e, por fim, docência $(2,7)$.

A estrutura curricular é organizada por disciplinas $(n=88 ; 93,6 \%)$ e em ciclos - básico, com disciplinas mais amplas ligadas às Ciências da Saúde, e profissionalizante, com disciplinas mais específicas ligadas à profissão $(n=58 ; 61,7 \%)$. Apesar disso, do total de cursos, 73 (77,7\%) contemplam atividades integradas e compartilhadas; desses, 59 são privados.

Em relação à carga horária total, a média computada foi de 5.364 horas, sendo 2.491 despendidas em conteúdos teóricos, 1.967 em conteúdos teórico-práticos e 907 em estágios/internatos. Entre os privados a média geral foi de 5.579 horas e, entre os públicos, de 5.148 horas.

Em relação aos locais onde os alunos realizam aulas práticas e/ou estágios, verificou-se que os cenários são múltiplos, sendo que todos os cursos utilizam unidades de atenção básica e hospitais gerais (Tabela 2). Questionados sobre os atores que acompanham os discentes nos cenários, citaram-se: o próprio docente $(n=93 ; 98,9 \%)$, profissionais da saúde $(n=60 ; 63,8 \%)$, e preceptores
Tabela 2. Cursos de graduação em enfermagem segundo utilização de cenários de atividades práticas e de estágios. Brasil, 2015-2016 ( $\mathrm{n=94).}$

\begin{tabular}{|c|c|c|}
\hline Cenário & $\mathbf{n}$ & $\%$ \\
\hline Unidades de Atenção Primária & 94 & 100 \\
\hline Hospitais gerais & 94 & 100 \\
\hline $\begin{array}{l}\text { Centro de Atenção Psicossocial } \\
\text { (CAPS) adulto }\end{array}$ & 85 & 90,4 \\
\hline Hospitais especializados & 83 & 88,3 \\
\hline $\begin{array}{l}\text { Núcleo de Apoio à Saúde da } \\
\text { Família (NASF) }\end{array}$ & 80 & 85,1 \\
\hline Instituições asilares de idosos & 80 & 85,1 \\
\hline $\begin{array}{l}\text { Unidades de Pronto Atendimento } \\
\text { (UPA) }\end{array}$ & 77 & 81,9 \\
\hline Creches e/ou escolas & 67 & 71,3 \\
\hline Policlínicas/ambulatórios/clínicas & 67 & 71,3 \\
\hline $\begin{array}{l}\text { Instituições para pessoas com } \\
\text { deficiência }\end{array}$ & 61 & 64,9 \\
\hline Empresas & 51 & 54,3 \\
\hline Residência Terapêutica & 37 & 39,4 \\
\hline Outros* & 07 & 7,4 \\
\hline
\end{tabular}

contratados $(n=42 ; 44,7 \%)$. Esta última estratégia é utilizada majoritariamente pelas instituições privadas $(\mathrm{n}=34)$.

\section{Perfil de competências dos egressos}

Com base no cálculo das médias ponderadas $\left(\bar{x}_{\mathrm{w}}\right)$ expostas na Tabela 3 , identifica-se que os cursos preparam os alunos de enfermagem para realizar a maioria das ações de saúde pesquisadas $\left(\bar{x}_{\mathrm{w}}=2,73\right)$, principalmente as de promoção e educação em saúde e a avaliação antropométrica; as menos trabalhadas são orientações de saúde bucal, pré-natal de alto risco e o atendimento de urgências e emergências psiquiátricas.

Em relação às ações gerenciais $\left(\bar{x}_{\mathrm{w}}=2,86\right)$, a maioria dos entrevistados considerou que o curso prepara os alunos para executar todas as ações listadas, exceto atestar óbito. Em se tratando dos procedimentos técnicos $\left(\bar{x}_{\mathrm{w}}=2,35\right)$, mais de $95 \%$ dos gestores concordaram que o curso prepara os egressos para realizar curativos, inserir sondas nasogástricas, nasoentéricas e uretrais, administrar medicamentos e vacinas. Os procedimentos atribuídos aos profissionais médicos apresentaram menores percentuais de concordância quan- 
Tabela 3. Médias ponderadas calculadas para as competências e habilidades avaliadas pelos respondentes quanto ao que o curso de graduação prepara os alunos para executá-las. Brasil, 2015-2016 $(\mathrm{n}=94)$.

\begin{tabular}{|c|c|}
\hline \multicolumn{2}{|l|}{ Competências verificadas } \\
\hline Ações de saúde & 2,73 \\
\hline Realizar ações de promoção da saúde & 2,98 \\
\hline Realizar grupos de educação em saúde & 2,98 \\
\hline Realizar avaliação antropométrica & 2,96 \\
\hline Trabalhar em equipe multidisciplinar & 2,94 \\
\hline Realizar consulta de puericultura & 2,94 \\
\hline Realizar pré-natal de baixo risco & 2,93 \\
\hline Realizar planejamento familiar & 2,87 \\
\hline $\begin{array}{l}\text { Realizar acolhimento de classificação de } \\
\text { risco }\end{array}$ & 2,86 \\
\hline $\begin{array}{l}\text { Realizar acompanhamento de pacientes } \\
\text { psiquiátricos }\end{array}$ & 2,67 \\
\hline $\begin{array}{l}\text { Realizar atendimento de urgência e } \\
\text { emergência psiquiátricas }\end{array}$ & 2,44 \\
\hline Realizar pré-natal de alto risco & 2,21 \\
\hline Realizar orientações de saúde bucal & 2,04 \\
\hline Ações gerenciais & 2,86 \\
\hline Realizar notificação compulsória & 2,93 \\
\hline Planejar e realizar campanha de vacinação & 2,92 \\
\hline Gerenciar Central de Esterilização Materiais & 2,87 \\
\hline Gerenciar Rede de Frio & 2,80 \\
\hline Supervisionar outros profissionais & 2,80 \\
\hline Atestar óbito & 1,30 \\
\hline Procedimentos técnicos & 2,35 \\
\hline Inserir sondas nasogástricas & 2,96 \\
\hline Realizar curativos & 2,96 \\
\hline Administrar medicamentos & 2,94 \\
\hline Administrar vacinas & 2,92 \\
\hline Inserir sondas uretrais & 2,90 \\
\hline Inserir sondas nasoentéricas & 2,88 \\
\hline Prescrever curativos & 2,76 \\
\hline Retirar pontos & 2,72 \\
\hline Inserir cateter venoso superficial & 2,69 \\
\hline Realizar punção arterial & 2,61 \\
\hline Realizar imobilizações & 2,49 \\
\hline Realizar parto normal emergencial & 2,01 \\
\hline Realizar parto normal & 1,98 \\
\hline Realizar drenagem de abscesso & 1,97 \\
\hline Inserir cateter venoso profundo & 1,56 \\
\hline Realizar Intubação traqueal & 1,45 \\
\hline Realizar sutura & 1,32 \\
\hline Realizar anestesia local & 1,26 \\
\hline
\end{tabular}

to ao preparo dos alunos para executá-los, em especial a anestesia local e sutura.
Tabela 3. Médias ponderadas calculadas para as competências e habilidades avaliadas pelos respondentes quanto ao que o curso de graduação prepara os alunos para executá-las. Brasil, 2015-2016 $(\mathrm{n}=94)$.

\begin{tabular}{|c|c|}
\hline Competências verificadas & \\
\hline $\begin{array}{l}\text { Procedimentos de apoio diagnóstico e } \\
\text { prescrições }\end{array}$ & 2,33 \\
\hline Realizar Papanicolau & 2,97 \\
\hline Interpretar resultado de Papanicolau & 2,88 \\
\hline $\begin{array}{l}\text { Interpretar resultado de teste tuberculínico } \\
\text { (PPD) }\end{array}$ & 2,79 \\
\hline Realizar teste do pezinho & 2,78 \\
\hline Realizar teste tuberculínico (PPD) & 2,78 \\
\hline Realizar eletrocardiograma & 2,77 \\
\hline Interpretar exames laboratoriais & 2,68 \\
\hline Interpretar exames de imagem & 2,41 \\
\hline Interpretar eletrocardiograma & 2,40 \\
\hline Solicitar exames laboratoriais & 2,28 \\
\hline Solicitar eletrocardiograma & 2,19 \\
\hline $\begin{array}{l}\text { Comunicar diagnóstico nosológico a } \\
\text { pacientes }\end{array}$ & 2,12 \\
\hline Tratar deficiências nutricionais & 2,11 \\
\hline Renovar prescrição médica & 2,08 \\
\hline Solicitar exames de imagem & 2,07 \\
\hline Prescrever anti-helmínticos & 2,04 \\
\hline $\begin{array}{l}\text { Prescrever medicamentos para casos de } \\
\text { doença crônica já diagnosticada }\end{array}$ & 2,03 \\
\hline Prescrever anti-inflamatórios & 2,01 \\
\hline Prescrever antifúngicos & 1,99 \\
\hline Prescrever medicamentos psicoativos & 1,98 \\
\hline $\begin{array}{l}\text { Prescrever medicamentos para casos de } \\
\text { insuficiência respiratória aguda }\end{array}$ & 1,95 \\
\hline Prescrever antibióticos & 1,94 \\
\hline
\end{tabular}

*Valores de 1 (discordância) a 3 (concordância).

Fonte: Dados da pesquisa.

Entre os procedimentos de apoio diagnóstico e prescrições $\left(\bar{x}_{w}=2,33\right)$, destacaram-se: realizar e interpretar resultado de Papanicolau, realizar eletrocardiograma e realizar e interpretar o teste tuberculínico. A prescrição de medicamentos e solicitação de exames de imagem, por outro lado, obtiveram menores médias.

A concordância de resposta entre os avaliadores para o conjunto das ações de saúde foi de apenas $30,3 \%\left(\mathrm{~W}_{\mathrm{a}}=0,303 ; \mathrm{p}=0,000\right)$, próximo aos valores verificados para os grupos de cursos privados, públicos e por regiões, exceto para a Centro-Oeste, cuja concordância foi superior a 50\%. Apenas entre os respondentes da região Norte não houve significância estatística, sugerindo 
Tabela 4. Percentual de concordância entre os entrevistados quanto ao preparo dos alunos durante a graduação para o desenvolvimento de competências e habilidades específicas, segundo grupamento de respondentes. Brasil, 2015-2016 ( $\mathrm{n}=94)$.

\begin{tabular}{|c|c|c|c|c|}
\hline \multirow[b]{2}{*}{$\begin{array}{l}\text { Grupamentos de } \\
\text { respondentes }\end{array}$} & \multicolumn{4}{|c|}{ Percentual de concordância $(\%)^{*}$} \\
\hline & Ações de saúde & Ações gerenciais & $\begin{array}{l}\text { Procedimentos } \\
\text { técnicos }\end{array}$ & $\begin{array}{l}\text { Apoio diagnóstico } \\
\text { e prescrições }\end{array}$ \\
\hline \multicolumn{5}{|l|}{ Esfera administrativa } \\
\hline Público & 29,3 & 54,2 & 46,2 & 46,2 \\
\hline Privado & 31,6 & 76,7 & 54,7 & 54,7 \\
\hline \multicolumn{5}{|l|}{ Regiões } \\
\hline Norte & $30,3^{\#}$ & 100 & 53,1 & 57,3 \\
\hline Nordeste & 25,4 & 61,9 & 60,9 & 47,9 \\
\hline Sudeste & 31,8 & 74,8 & 54,4 & 53,5 \\
\hline Sul & 33,6 & 69,0 & 53,4 & 61,5 \\
\hline Centro-Oeste & 54,8 & 68,1 & 53,0 & 45,9 \\
\hline Total da amostra & 30,3 & 65,4 & 70,5 & 51,8 \\
\hline
\end{tabular}

${ }^{\star}$ Coeficiente de concordância de Kendall; ${ }^{*} \mathrm{p}>0,005$.

Fonte: Dados da pesquisa.

que estes respondentes, em específico, discordam significativamente no que concerne ao preparo do aluno, o que remete a realidades de formação muito específicas entre os cursos. Outros resultados podem ser observados na Tabela 4 .

\section{Discussão}

Neste estudo, o perfil dos gestores de cursos de graduação em enfermagem entrevistados, sendo 95\% deles enfermeiros, confirma os dados encontrados no âmbito da pesquisa nacional Perfil da Enfermagem. Segundo a pesquisa, $85,1 \%$ do total de 1.804 .535 profissionais de enfermagem são mulheres e $40,1 \%$ têm idades entre $36-50$ anos, faixa etária denominada, com base na sociologia das profissões, de fase da "maturidade profissional". Esta fase inclui os profissionais de enfermagem que, em sua maioria, já estão em pleno desenvolvimento de suas capacidades profissionais e possuem diploma de pós-graduação ${ }^{12}$.

Quanto aos cursos onde atuam os respondentes, sua distribuição e concentração regional acompanha o panorama nacional do total de cursos de enfermagem, do total de cursos da área da saúde e, de modo mais abrangente, do total de cursos de todas as áreas ${ }^{10}$. Os dados confirmam a hegemonia da região Sudeste como celeiro de formação e retenção profissional, já que, do total de enfermeiros inscritos nos conselhos regionais da profissão, $46,1 \%$ se formaram nos estados de São Paulo, Rio de Janeiro e Minhas Gerais e $48,2 \%$ atuam nessa região ${ }^{12}$.
A proporção de cursos privados incluídos na amostra também se justifica no cenário brasileiro, que apresenta maior número de cursos de instituições de ensino administradas por esta esfera jurídica, cuja proporção aumentou exponencialmente no fim da década de 1990, pós-promulgação da Lei de Diretrizes e Bases de $1996^{13}$. Esta esfera, em 1991, era responsável por 46 cursos de enfermagem; em 2016 passou a 791, equivalente a $82,2 \%$ do total de cursos de enfermagem ${ }^{14}$. Deste panorama reflete o fato de que $57,4 \%$ dos atuais enfermeiros do Brasil se formaram em instituições privadas ${ }^{12}$.

Outra repercussão da proliferação de cursos de enfermagem foi o aumento da oferta de vagas desproporcional à procura por eles, que se confirma pelo aumento progressivo da taxa de ociosidade. Em 2004, esta taxa era de $15,8 \%$, passando a $62,9 \%$ em 2016, correspondente a mais de 158 mil vagas não preenchidas, das quais cerca de 155 mil são de cursos privados ${ }^{14}$.

As correntes desigualdades de adequação entre a disponibilidade e a demanda de vagas são devidas, muito em parte, à pouca regulação sobre o sistema formador e à dissociação que persiste entre a oferta de formação e as necessidades de recursos humanos para o SUS ${ }^{15}$. Esse descompasso incorre em distorção no preparo profissional ao enfatizar um treinamento que pouco favorece o sistema de saúde ${ }^{16}$, de modo que estabelecer uma articulação entre as instituições formadoras e o SUS tem sido um desafio persistente ${ }^{17,18}$.

É neste contexto que se inserem as DCENF como guia condutor da formação de enferma- 
gem, que ensejam um egresso que atue como agente de mudança, capaz de subsidiar a transformação dos problemas de saúde da população brasileira ${ }^{7}$. Foi com base nessa premissa que este estudo buscou identificar elementos característicos da estrutura curricular e do perfil de egressos de cursos de graduação em enfermagem que se aproximem dos pressupostos das DCENF e da atuação do enfermeiro na atenção básica, nível de atenção prioritário e ordenador do SUS.

Constatou-se que, segundo os entrevistados, a maior ênfase do processo formativo do enfermeiro é a atenção básica, diferente do encontrado por estudo que entrevistou em profundidade gestores de ensino de diferentes regiões do Brasil. Segundo o referido estudo, os cursos apresentam ênfases plurais, mas que tendem sobretudo para o nível terciário ou nível básico de atenção ${ }^{19}$.

Também se verificou que os currículos se organizam por disciplinas distribuídas nos ciclos básico e profissionalizante, assim como apurado por estudos prévios ${ }^{19-21}$. Essa característica remonta a proposta flexneriana de fragmentação do ensino médico - adotada por todos os cursos da saúde -, que defende o domínio da teoria, no ciclo básico, para posterior entendimento da prática, no ciclo profissionalizante. Historicamente estes ciclos se encontram desassociados, de modo que o que é ministrado no primeiro possui pouca aplicabilidade prática no segundo. Assim, a manutenção desse modelo de estrutura curricular revela um ainda descompasso ao proposto pelas DCENF $^{19,22}$.

Grande parte dos cursos, em especial os privados, contempla atividades integradas com outros cursos, característica que pode estar fundamentada na ascensão da proposta de Educação Interprofissional (EIP), apontada como premissa para reorientação do modelo de formação e de atenção à saúde ${ }^{23}$. Por outro lado, como esta questão do questionário não solicitou detalhamento, as respostas podem estar relacionadas apenas ao fato de que, na maioria dos cursos privados, as disciplinas básicas (períodos iniciais) são ministradas em conjunto para diversos cursos da saúde. Isto não implica necessariamente o desenvolvimento dos pressupostos da interprofissionalidade nem, portanto, o desenvolvimento de competências para o trabalho em equipe.

Em relação à carga horária total dos cursos, a média computada é superior à mínima de 4 mil horas exigida pelo Conselho Nacional de Educação ${ }^{24}$. De igual maneira, o tempo médio destinado a estágios/internatos é também maior às 500 horas exigidas como mínimas pelas DCENF7.
Sobre as atividades práticas, os cursos inserem seus estudantes em diferentes dispositivos de saúde e espaços comunitários, em concordância com o que propõem as $\mathrm{DCENF}^{7}$. Essa diversificação dos cenários para além do hospital sugere o entendimento do conceito ampliado de saúde, na contramão do processo de formação historicamente hegemônico, que tem como foco de cuidado a doença e o hospital como lócus privilegiado de cura e ensino ${ }^{21}$. Sugere, ainda, uma aproximação entre o mundo do ensino e o do trabalho, tão necessária ao desenvolvimento de competências que permitam ao profissional intervir no perfil epidemiológico, contribuindo, assim, para a melhoria da qualidade de vida das populações ${ }^{20,25,26}$.

Outros estudos já apontam para esta realidade, porém, muitas são as dificuldades que permeiam a introdução dos estudantes na realidade do SUS, como a resistência, despreparo e número insuficiente de docentes e preceptores, infraestrutura precária das unidades e até mesmo a disputa de campos por diferentes instituiçõos ${ }^{9,19,27}$.

Nessa ótica, para que esses cenários favoreçam a formação de enfermeiros mais conscientes do seu papel social, é preciso investir, minimamente, na qualificação das redes de atenção e dos profissionais que acompanham alunos, e na contratualização de objetivos e metas entre as instituições de ensino e os serviços do SUS. Mais ainda, é preciso enfrentar a herança das políticas, práticas e ensino de saúde verticais e fragmen$\operatorname{tadas}^{28}$, em prol da integralidade e qualidade da atenção à saúde e do desenvolvimento de competências que permitam ao egresso diagnosticar e solucionar problemas de saúde de diferentes complexidades $^{7,9}$.

$\mathrm{Na}$ atenção básica, as demandas são plurais, porém concentradas em poucos problemas de saúde de cunho social, em enfermidades benignas comuns em uma comunidade e crônicas não transmissíveis passíveis de controle. Assim, a atuação profissional nesse nível de atenção requer o uso de tecnologias de alta complexidade e baixa densidade, isto é, exige profundo conhecimento e aportes técnicos generalistas, apoiados por procedimentos mais simples e menos onerosos ao sistema de saúde ${ }^{8,29}$.

Considerando o exposto, questionou-se sobre o preparo dos estudantes para o desenvolvimento de ações de saúde comuns. A análise apontou para um perfil profissional apto a desenvolver principalmente ações de promoção e educação em saúde, gerenciais e técnicas. Esta apreensão foi confirmada estatisticamente pela existência de concordância, embora baixa, entre os respon- 
dentes, sugerindo que os processos formativos dos diferentes cursos se assemelham.

$\mathrm{Na}$ atenção básica, em conformidade com o modelo de saúde preconizado pelo SUS, as ações educativas têm papel basilar e permeiam toda a assistência da enfermagem, sendo necessário um processo formativo que os aproxime dessa perspectiva ${ }^{30,31}$. Também destacadas, as competências administrativas e gerenciais são preconizadas pelas DCENF e são características do trabalho do enfermeiro, que se mostra mais preparado para assumir tais funções ${ }^{32}$.

A assistência pouco invasiva a diferentes grupos populacionais, a exemplo do preventivo ginecológico, puericultura, avaliação antropométrica e pré-natal de baixo risco, também é competência bastante trabalhada durante o curso, ratificando a atenção à saúde da criança $\mathrm{e}$ da mulher como prioritárias no campo da saúde pública. Estudos encontraram que essas práticas são as mais comumente desenvolvidas por enfermeiros da atenção básica ${ }^{33,34}$, confirmando que a atuação desse profissional foi e é fundamental para a implementação das políticas voltadas a estas populações, que historicamente resultaram na redução de mortalidade materno-infantil ${ }^{35}$.

Quanto às atribuições de solicitar exames complementares e de prescrever medicamentos conforme protocolos assistenciais, embora previstas pela legislação que regulamenta a profissão de enfermagem ${ }^{36}$ e pela Política Nacional de Atenção Básica ${ }^{37}$, elas são menos enfatizadas durante a graduação. Essa perspectiva não revela surpresa, dado que, no Brasil, ainda há muitas controvérsias sobre as bases legais e éticas para a realização dessas atribuições por enfermeiros. $\mathrm{E}$ apesar desse cenário, a implementação de práticas avançadas para a enfermagem já vem sendo considerada no País ${ }^{38}$.

Esta estratégia tem sido introduzida em diferentes países a fim de melhorar a acessibilidade aos cuidados primários de saúde, com base em resultados de pesquisas que demonstram que ela fornece cuidados análogos ou mais eficazes aos cuidados prestados por médicos ${ }^{39-41}$.

No Brasil, a discussão sobre o tema é especialmente motivada pela escassez de médicos no âmbito da atenção básica, sobretudo em locais de difícil acesso, o que repercute diretamente na ineficiência e pouca resolutividade desse nível de atenção ${ }^{42,43}$. Diante desta perspectiva, outras evidências sobre a formação de enfermagem precisam ser produzidas e exploradas, tendo como horizonte um cenário de implementação de funções avançadas para os enfermeiros.

\section{Conclusões}

Esta pesquisa buscou apresentar um panorama da oferta e das características de formação de enfermagem no Brasil, identificando elementos consoantes às DCENF e às competências requeridas para a atuação do enfermeiro na atenção básica.

Verificou-se oferta de vagas distante das necessidades nacionais e que a estrutura curricular dos cursos se centra em disciplinas fragmentadas nos ciclos básico e profissionalizante, destoando do recomendado pelas DCENF e pela perspectiva de integralidade tão cara ao modelo de atenção do SUS.

Quanto ao perfil de competências, a formação prepara os alunos principalmente para o desenvolvimento de ações de promoção, prevenção, gerenciamento e técnicas de enfermagem, que além de previstas no escopo de competências e habilidades gerais preconizadas para o exercício profissional generalista da enfermagem, são compatíveis com as principais demandas assistenciais da atenção básica. Por outro lado, práticas legalmente permitidas ao enfermeiro, mas historicamente compreendidas como exclusivas dos médicos, são menos ressaltadas, a exemplo da solicitação de exames e prescrição medicamentosa para doenças comuns.

Apesar de a pesquisa não ter alcançado 100\% da amostra pretendida, considera-se que o estudo foi capaz de fornecer dados para a descrição do panorama nacional da formação de enfermagem, resguardadas as precauções de generalização dos dados.

Pelo exposto, recomenda-se: maior intervenção do Estado no que diz respeito à abertura de vagas sem uma avaliação prévia das necessidades locais; estabelecimento de um processo de revisão das DCENF, numa tentativa de definir competências específicas que possam dar conta das principais demandas do SUS; e novas investigações que conjuguem formação, prática e legislação profissional. 


\section{Colaboradores}

C Magnago participou da concepção e delineamento, análise e interpretação dos dados, redação do artigo e aprovação da versão a ser publicada. CR Pierantoni participou da concepção e delineamento, revisão crítica e aprovação da versão a ser publicada.

\section{Referências}

1. Bellaguarda MLR, Padilha MI, Pereira Neto AF, Pires D, Peres MAA. Reflexão sobre a legitimidade da autonomia da enfermagem no campo das profissões de saúde à luz das ideias de Eliot Freidson. Esc Anna Nery 2013; 17(2):369-374.

2. Magnago C, Tavares CMM. A formação de enfermagem direcionada para os ideais da reforma psiquiátrica. Enferm Brasil 2011; 10(2):99-107.

3. Capella BB, Faria EM, Gelbcke FL, Spricigo JS. Profissionalização da enfermagem: uma necessidade social. Rev Bras Enferm 1988; 41(2):161-168.

4. Germano RM. Educação e ideologia da Enfermagem no Brasil (1955-1980). São Caetano do Sul (SP): Yendis; 2007.

5. Cordeiro H. A indústria da saúde no Brasil. Rio de Janeiro: Graal; 1980.

6. Lima MDS. Ensino de enfermagem: retrospectiva, situação atual e perspectivas. Rev Bras Enferm 1994; 47(3):270-277.

7. Ministério da Educação (MEC). Conselho Nacional de Educação (CNE). Parecer CNE/CES no 1.133 de 7 de agosto de 2001. Diretrizes Curriculares Nacionais dos Cursos de Graduação em Enfermagem, Medicina e Nutrição. Diário Oficial da União 2001; 1 out.

8. Mendes EV. A construção social da Atenção Primária à Saúde. Brasília: Conselho Nacional de Secretários de Saúde; 2015.

9. Peres CRFB, Marin MJS, Soriano ECI, Ferreira MLSM. Um olhar dialético para as mudanças curriculares na formação do enfermeiro. Rev Esc Enferm USP 2018; 52:e03397.

10. Brasil. Ministério da Educação (MEC). Instituto Nacional de Estudos e Pesquisas Educacionais Anísio Teixeira (INEP). Censo da Educação Superior 2013. Brasília: INEP/MEC; 2014.

11. Conselho Federal de Enfermagem (Cofen). Resoluções [página na Internet]. 2016 [acessado 2016 Abr 15]. Disponível em: http://www.cofen.gov.br/categoria/ legislacao/resolucoes

12. Machado $\mathrm{MH}$, Wermelinger M, Vieira M, Oliveira E, Lemos W, Aguiar Filho W, Lacerda WF, Santos MR, Souza Junior PB, Justino E, Barbosa C. Aspectos gerais da formação da enfermagem: o perfil da formação dos enfermeiros, técnicos e auxiliares. Enferm Foco 2016; 6(2):15-34.

13. Maas LWD. Análise comparativa da base social da Medicina e Enfermagem no Brasil entre os anos de 2000 e 2010. Cad Saude Publica 2018; 34(3):e00199116.

14. Rede Observatório de Recursos Humanos em Saúde - ObservaRH. Estação de Trabalho Instituto de Medicina Social da Universidade do Estado do Rio de Janeiro. Sistema de Indicadores das Graduações em saúde - SIGRAS [página na Internet]. 2014 [acessado 2014 Mar 03]. Disponível em: http://www.obsnetims.org. br/sigras/

15. Dal Poz MR, Perantoni CR, Girardi S. Formação, mercado de trabalho e regulação da força de trabalho em saúde no Brasil. In: Fundação Oswaldo Cruz (Fiocruz). A saúde no Brasil em 2030: prospecção estratégica do sistema de saúde brasileiro: organização e gestão do sistema de saúde. Rio de Janeiro: Fiocruz; 2013. p. 187-233. 
16. Pierantoni R, Magnago C. Tendências das graduações em Saúde no Brasil: análise da oferta no contexto do Mercosul. Divulg Saude Debate 2017; 57:30-43.

17. França T, Magnago C, Santos MR, Belisário SA, Silva CBG. PET-Saúde/GraduaSUS: retrospectiva, diferenciais e panorama de distribuição dos projetos. Saúde Debate 2018; 42(n. esp. 2):286-301.

18. Dias HS, Lima LD, Teixeira M. A trajetória da política nacional de reorientação da formação profissional em saúde no SUS. Cien Saude Colet 2013; 18(6):16131624.

19. Magnago C, Pierantoni CR, Carvalho CL, Girardi $\mathrm{SN}$. Nurse training in health in different regions in Brazil. Rev Bras Saude Mater Infant 2017; 17(Supl. 1):S219-S228.

20. Silva MJ, Sousa EM, Freitas CL. Formação em enfermagem: interface entre as diretrizes curriculares e os conteúdos de atenção básica. Rev Bras Enferm 2011; 64(2):315-321.

21. Peres CRFB, Marin MJS, Tonhom SFR, Barbosa PMK. Teaching-service's integration in the training of nurses in the state of São Paulo (Brazil). Rev Min Enferm 2018; 22:e-1131.

22. Moura DTD, Foltran RS, Fraiz IC, Novak EM. Articulação Entre os Ciclos Básico e Profissionalizante: percepção dos alunos da UFPR. Rev Bras Educ Med 2018; 42(1):226-236.

23. Frenk J, Chen L, Bhutta ZA, Cohen J, Crisp N, Evans T, Fineberg H, Garcia P, Ke Y, Kelley P, Kistnasamy B, Meleis A, Naylor D, Pablos-Mendez A, Reddy S, Scrimshaw S, Sepulveda J, Serwadda D, Zurayk H. Health professionals for a new century: transforming education to strengthen health systems in an interdependent world. Lancet 2010; 376(9756):1923-1958.

24. Brasil. Ministério da Educação (MEC). Conselho Nacional de Educação (CNE). Câmara de Educação Superior (CES). Resolução CNE/CES no 4 de 6 de abril de 2009. Dispõe sobre carga horária mínima e procedimentos relativos à integralização e duração dos cursos de graduação em Biomedicina, Ciências Biológicas, Educação Física, Enfermagem, Farmácia, Fisioterapia, Fonoaudiologia, Nutrição e Terapia Ocupacional, bacharelados, na modalidade presencial. Diário Oficial da União 2009; 7 abr.

25. Fernandes JD, Rebouças LC. Uma década de Diretrizes Curriculares Nacionais para a Graduação em Enfermagem: avanços e desafios. Rev Bras Enferm 2013; 66(n. esp.):95-101.

26. Santos SSC. Perfil de egresso de Curso de Enfermagem nas Diretrizes Curriculares Nacionais: uma aproximação. Rev Bras Enferm 2006; 59(2):217-221.

27. Vieira SP, Pierantoni CR, Magnago C, Ney MS, Miranda RG. A graduação em medicina no Brasil ante os desafios da formação para a Atenção Primária à Saúde. Saude Debate 2018; 42(n. esp. 1):189-207.

28. Reis AAC, Sóter APM, Furtado LAC, Pereira SSS. Thoughts on the development of active regional public health systems. Cien Saúde Colet 2017; 22(4):10451054.

29. Pimentel IRS. Caracterização da demanda em uma Unidade de Saúde da Família. Rev Bras Med Fam Comunidade 2011; 6(20):175-181.
30. Simon E, Jezine E, Vasconcelos EM, Ribeiro KSQS. Metodologias ativas de ensino-aprendizagem e educação popular: encontros e desencontros no contexto da formação dos profissionais de saúde. Interface (Botucatu) 2014; 18(2):1355-1364.

31. Leonello VM, Vieira MPM, Duarte TCR. Competencies for educational actions of Family Health Strategy nurses. Rev Bras Enferm 2018; 71(3):1072-1078.

32. Coatto A, Traldi MC. Competências gerenciais do enfermeiro na atenção primária. Rev Saude Colet 2011; 8(52):180-186.

33. Ermel RC, Fracolli LA. O trabalho das enfermeiras no Programa de Saúde da Família em Marília/SP. Rev Esc Enferm USP 2006; 40(4):533-539.

34. Matumoto S, Vieira KCS, Pereira MJB, Santos CB, Fortuna CM, Mishima SM. Production of nursing care in primary health care services. Rev Lat Am Enfermagem 2012; 20(4):710-717.

35. Branquinho ID, Lanza FM. Child health in Primary Care: Evolution of Brazilian policies and nurses' performance. RECOM 2018; 8:e2753.

36. Brasil. Lei no 7.498 de 25 de junho de 1986. Dispõe sobre a regulamentação do exercício da enfermagem, e dá outras providências. Diário Oficial da União 1986; 26 jun.

37. Brasil. Ministério da Saúde (MS). Secretaria de Atenção à Saúde. Departamento de Atenção Básica. Política Nacional de Atenção Básica. Brasília: MS; 2012.

38. Martiniano CS, Coelho AA, Latter S, Uchôa SAC. Medication prescription by nurses and the case of the Brazil: what can we learn from international research? Int J Nurs Stud 2014; 54(8):1071-1073.

39. Dierick-van Daele AT, Metsemakers JF, Derckx EW, Spreeuwenberg C, Vrijhoef HJ. Nurse practitioners substituting for general practitioners: randomized controlled trial. J Adv Nurs 2009; 65(2):391-401.

40. Mundinger MO, Kane RL, Lenz ER, Totten AM, Tsai WY, Cleary PD, Friedewald WT, Siu AL, Shelanski ML. Primary care outcomes in patients treated by nurse practitioners or physicians: a randomized trial. JAMA 2000; 283(1):59-68.

41. Cassiani SLB, Rosales LK. Initiatives towards Advanced Practice Nursing Implementation in the Region of the Americas. Esc Anna Nery 2016; 20(4):e20160081.

42. Girardi SN, coordenador. Relatório Final: Identificação de áreas de escassez em recursos humanos em saúde no Brasil. Belo Horizonte: EPSM, NESCON, UFMG; 2012.

43. Seale C, Anderson E, Kinnersley P. Treatment advice in primary care: a comparative study of nurse practitioners and general practitioners. J Adv Nurs 2006; 54(5):534-541.

Artigo apresentado em 30/04/2019

Aprovado em 20/08/2019

Versão final apresentada em 30/09/2019 